\title{
Comparison of outcomes following laparoscopic and open hysterectomy with pelvic lymphadenectomy for early stage endometrial carcinoma
}

\author{
Xu Cong $\underline{R u a n}^{1}, \mathrm{MD}, \mathrm{BSc}(\mathrm{Hons})$, Wai Loong Wong $^{2}, \mathrm{MRCOG}$ MMed, Hui Qing Yeong ${ }^{1}$, MD, BEng,
} Yong Kuei Timothy $\underline{\mathrm{im}}^{2}$, MRCOG, FAMS

\begin{abstract}
INTRODUCTION Endometrial carcinoma is the most common gynaecological malignancy. Studies have shown that laparoscopic total hysterectomy, bilateral salpingo-oophorectomy and pelvic lymph node dissection was advantageous compared to laparotomy in reducing length of stay and intraoperative blood loss. However, these studies had a predominantly Caucasian population. A comparison study was conducted among the Singapore population to investigate the differences in oncological and surgical outcomes between these two methods.

METHODS A retrospective, single-centre cohort study was conducted. Records of hospitalised patients with Stage 1 endometrioid carcinoma from 2008 to 2014 were extracted for review. Demographic data and study-specific parameters, including operative time, length of hospitalisation, intraoperative and postoperative complications, pain scores, final staging and recurrence rates, were compared between the two groups.

RESULTS 475 endometrioid carcinoma patients were admitted for surgical staging, among whom 374 fulfilled our inclusion criteria. Out of these patients, 229 underwent laparotomy and 145 underwent laparoscopy. The race, parity and body mass index of both groups were comparable. Patients who underwent laparoscopic surgery reported reduced pain score within two hours postoperatively $(p=0.007)$ and at Postoperative Days 1,2 and $3(p<0.001)$. Laparoscopic surgery also illustrated better outcomes such as reduced length of stay $(p<0.001)$ and reduced intraoperative blood loss $(p<0.001)$. The operative time, recurrence rate and disease-free intervals were comparable between both groups. CONCLUSION Laparoscopy offered similar oncological outcomes with superior surgical outcomes compared to laparotomy. It provides a suitable alternative in the surgical staging of endometrioid carcinoma.
\end{abstract}

Keywords: endometrial carcinoma, hysterectomy, laparoscopy, laparotomy

\section{INTRODUCTION}

Endometrial carcinoma is the most commonly diagnosed gynaecological malignancy. ${ }^{(1)}$ It occurs predominantly in postmenopausal women and is associated with significant morbidity and mortality. ${ }^{(2)}$ The main form of treatment for early stage endometrial carcinoma is total hysterectomy, bilateral salpingo-oophorectomy (THBSO) and pelvic lymph node dissection (PLND). ${ }^{(2)}$ Some patients may receive para-aortic lymphadenectomy, depending on their tumour grade and tumour spread to the lymph nodes. ${ }^{(3)}$ In the local context, para-aortic nodes are removed if malignant pelvic nodes are present, when the para-aortic nodes are clinically enlarged, and if there is the presence of high-grade tumour, deep myoinvasion and adnexal involvement. PLND includes lymphadenectomy of the lymph nodes around the lower portion of the common iliac, the external and internal iliac, and the obturator arteries bilaterally.

With the development of minimally invasive surgery, multiple studies have shown that women who are older or obese may benefit from laparoscopic surgery for early endometrial carcinoma. ${ }^{(4,5)}$ The benefits of laparoscopic surgery include shorter hospital stays, reduced pain score and fewer intraoperative or postoperative complications. ${ }^{(4-6)}$ However, given that this data came from a predominantly Caucasian population, we conducted an Asian study to investigate the utility of laparoscopy in improving surgical outcomes compared to laparotomy. This would help us to determine the best method to surgically stage patients with early stage endometrial carcinoma in the Singapore population.

\section{METHODS}

A retrospective, single-centre study was conducted at KK Women's and Children's Hospital, Singapore. We extracted for review the medical records of patients who were hospitalised from 2008 to 2014 with FIGO (International Federation of Gynecology and Obstetrics) surgical Stage 1 endometrioid adenocarcinoma of the uterus. Endometrial carcinoma was diagnosed after an endometrial biopsy or curettage. The inclusion criteria were: (a) tissue-proven endometrioid carcinoma; (b) standard surgical procedure consisting of THBSO and PLND; and (c) postoperative surgical staging consistent with Stage 1 endometrioid carcinoma. The tumour grade of the included patients ranged from Grade 1 to 3. Patients with synchronous carcinoma or mixed tumours were excluded. The patients were classified into two arms: laparoscopic and laparotomy THBSO with PLND. A sample size calculation was performed based on a similar study by Boggess et al. ${ }^{(7)}$ Based on their observed overall complication rate of

${ }^{1}$ Duke-NUS Medical School, 2Department of Gynaecological Oncology, KK Women's and Children's Hospital, Singapore

Correspondence: Dr Wong Wai Loong, Consultant, Department of Gynaecological Oncology, KK Women's and Children's Hospital, 100 Bukit Timah Road, Singapore 229899. wong.wai.loong@singhealth.com.sg 
$29.7 \%$ for laparotomy and $13.6 \%$ for laparoscopy, a sample size of 114 subjects in each group was required for sufficient power to rule out false negatives. A total of 475 patients were selected for the present study.

Data was retrieved from the hospital's electronic clinical data monitoring system and patients' medical case notes. The study was approved by the institution's Institutional Review Board with a waiver for patient consent. Information extracted from each patient's medical record included demographic data such as age, race, parity and body mass index. Study-specific parameters included operative time, length of hospitalisation, intraoperative and postoperative complications, estimated blood loss, pain scores on the day of the operation and Postoperative Days 1, 2 and 3, the number of lymph nodes dissected, final staging, type of adjuvant therapy and recurrence rates.

Pain scores were assessed using a numerical rating scale of $0-10$, where 0 signified no pain and 10 signified the worst pain that the patient could imagine. Operative time was defined as the time from skin incision to skin closure. All patients had their surgical drains secured after THBSO and PLND, and these drains were removed once there was no further drainage of serous fluid and blood, usually within three to four days. Drain output was considered prolonged if it was not removed within one week postoperatively. Implications of prolonged drain output include undiagnosed ureteral injury, intra-abdominal haemorrhage and inflammation. Blood loss was estimated using the difference in the total amount of suction fluid and irrigation fluid after the operation. Adjuvant therapy was tailored to the pathological findings that were noted postoperatively during the multidisciplinary tumour board discussion with the gynaecologic oncology, radiation oncology, medical oncology, radiology and pathology teams.

All analysis was performed using IBM SPSS Statistics version 21.0 (IBM Corp, Armonk, NY, USA). Descriptive statistics were used to describe the patients' characteristics. Continuous variables were compared using Student's t-test and categorical variables using Fisher's exact test. A p-value $<0.05$ was considered to be statistically significant.

\section{RESULTS}

Between 2008 and 2014, 475 patients were admitted for early stage endometrial carcinoma for surgical staging. A total of 101 patients were excluded due to mixed endometrioid tumour ( $n=8)$, modified surgeries $(n=64)$ or synchronous tumours $(n=29)$. Out of the 374 included patients, 229 underwent laparotomy and 145 underwent laparoscopic surgery. Five patients among the 145 laparoscopic surgery patients were converted to laparotomy intraoperatively due to inability to deliver the uterus $(n=1)$, bleeding pedicle $(n=1)$, dense adhesions $(n=2)$ or the anaesthetic complication of severe intraoperative bronchospasm $(n=1)$.

Patients who underwent laparotomy had a higher mean age compared to the laparoscopic surgery group (55.6 \pm 9.7 years vs. $53.0 \pm 11.0$ years; $p=0.015)$. The race, parity and body mass index of both groups were comparable (Table I). While the two procedures were comparable in terms of operation time, patients who underwent laparoscopic surgery reported a reduced pain score within two hours postoperatively $(p=0.007)$ and on Postoperative Day 1, 2 and 3 ( $p<0.001$ ). Laparoscopic surgery also had better outcomes, such as reduced length of stay $(p<0.001)$, postoperative complications $(p=0.002)$ and intraoperative blood loss $(p<0.001)$. No vascular complications or bowel injuries were reported during laparoscopic surgery.

Patients who underwent laparoscopic surgery had fewer lymph nodes removed compared to those who underwent laparotomy $(24.7 \pm 9.6$ vs. $27.3 \pm 10.7 ; p=0.016)$. However, there were no significant differences in terms of recurrence rate and disease-free intervals between both groups.

In terms of postoperative surgical site infection, significantly more patients suffered this complication in the laparotomy group compared to the laparoscopic surgery group ( $n=36$ vs. $n=6$; $p<0.001)$. The risk of lymphoedema, as monitored regularly through outpatient follow-ups, was comparable between both groups $(p=0.644)$. No re-interventions were required for either groups. Overall postoperative survival for both laparotomy and laparoscopic surgery was $100 \%$.

\section{DISCUSSION}

Our retrospective study confirmed that laparoscopy was as effective as laparotomy in terms of achieving comparable oncological outcomes in early stage endometrial carcinoma. In terms of intraoperative and postoperative complications, laparoscopy was comparable, and possibly superior, to laparotomy. Estimated blood loss and surgical site infection were significantly reduced in the laparoscopic group, likely secondary to smaller abdominal wall incisions. Postoperatively, pain scores were significantly reduced in the laparoscopic group when monitored from the day of the operation to Postoperative Day 4. This was comparable to other studies found in the literature. ${ }^{(3,8,9)}$ Laparoscopy had the advantage of decreasing the complication rate, blood loss and postoperative pain, and hence was associated with a shorter hospital stay, reduced need for analgesia and an early return to normal activities, thereby improving the quality of life of these patients. ${ }^{(10)}$ Given the high prevalence of diabetes mellitus in our population, a shorter length of stay would reduce the risk of postoperative infection and wound breakdown, ${ }^{(11)}$ thereby allowing for more efficient use of healthcare resources.

Operative time was found to be comparable for laparoscopy and laparotomy, which illustrated the increase in popularity of laparoscopy in the local population and surgeons' high comfort level in handling laparoscopic devices. The operative time, estimated blood loss and number of extracted lymph nodes in our study were comparable to findings in the international literature. ${ }^{(7,12,13)}$ While there was a reduction in the number of lymph nodes extracted through laparoscopy compared to laparotomy, pelvic lymphadenectomy was complete in both approaches. There was no difference in the recurrence rate of carcinoma in the two groups. As illustrated by our study and other international literature, laparoscopy provided similar oncological outcomes compared to laparotomy and the standard of care was not compromised in both approaches. ${ }^{(2,5,11)}$ 
Table I. Patient demographics and study parameters.

\begin{tabular}{|c|c|c|c|}
\hline \multirow[t]{2}{*}{ Parameter } & \multicolumn{2}{|c|}{ No. $(\%) / m e a n \pm$ standard deviation } & \multirow[t]{2}{*}{ p-value } \\
\hline & Laparotomy $(n=229)$ & Laparoscopy $(n=145)$ & \\
\hline Age (yr) & $55.6 \pm 9.7$ & $53.0 \pm 11.0$ & $0.015^{+}$ \\
\hline Ethnicity & & & 0.085 \\
\hline Chinese & $157(68.6)$ & $116(80.0)$ & \\
\hline Malay & $35(15.3)$ & $18(12.4)$ & \\
\hline Indian & $27(11.8)$ & $6(4.1)$ & \\
\hline Eurasian & $2(0.9)$ & $1(0.7)$ & \\
\hline Others & $8(3.5)$ & $4(2.8)$ & \\
\hline Parity & $1.8 \pm 1.6$ & $1.6 \pm 1.4$ & 0.132 \\
\hline BMI $\left(\mathbf{k g} / \mathbf{m}^{2}\right)$ & $28.7 \pm 6.9$ & $28.1 \pm 5.7$ & 0.348 \\
\hline Operation time (min) & $178.5 \pm 51.6$ & $187.2 \pm 41.8$ & 0.088 \\
\hline Length of stay (day) & $7.0 \pm 6.2$ & $4.7 \pm 2.5$ & $<0.001^{+}$ \\
\hline Blood loss (mL) & $155.5 \pm 237.8$ & $78.6 \pm 164.0$ & $<0.001^{+}$ \\
\hline \multicolumn{4}{|l|}{ Pain score } \\
\hline POD 0 & $1.9 \pm 1.5$ & $1.5 \pm 1.2$ & $0.007^{\dagger}$ \\
\hline POD 1 & $1.5 \pm 1.0$ & $1.1 \pm 0.8$ & $<0.001^{+}$ \\
\hline POD 2 & $1.1 \pm 1.0$ & $0.6 \pm 0.6$ & $<0.001^{+}$ \\
\hline POD 3 & $0.8 \pm 0.9$ & $0.3 \pm 0.5$ & $<0.001^{+}$ \\
\hline Complication & $92(40.2)$ & $35(24.1)$ & $0.002^{+}$ \\
\hline Lymphoedema & $33(14.4)$ & $18(12.4)$ & 0.644 \\
\hline Wound breakdown & $36(15.7)$ & $6(4.1)$ & $<0.001^{+}$ \\
\hline Vault haematoma & $4(1.7)$ & $3(2.1)$ & 1.000 \\
\hline Bleeding & $0(0)$ & $1(0.7)$ & 0.388 \\
\hline Seroma & $1(0.4)$ & $1(0.7)$ & 1.000 \\
\hline Lymphocyst & $3(1.3)$ & $2(1.4)$ & 1.000 \\
\hline Postoperative fever & $4(1.7)$ & $2(1.4)$ & 1.000 \\
\hline Limb weakness & $1(0.4)$ & $1(0.7)$ & 1.000 \\
\hline Prolonged drain output & $2(0.9)$ & $1(0.7)$ & 1.000 \\
\hline Sepsis & $1(0.4)$ & $0(0)$ & 1.000 \\
\hline Incisional hernia & $2(0.9)$ & $0(0)$ & 0.524 \\
\hline Drain site haematoma & $1(0.4)$ & $0(0)$ & 1.000 \\
\hline Limb paraesthesia & $2(0.9)$ & $0(0)$ & 0.524 \\
\hline Vein injury & $1(0.4)$ & $0(0)$ & 1.000 \\
\hline Bowel leakage & $1(0.4)$ & $0(0)$ & 1.000 \\
\hline Endometrial final stage* & & & 1.000 \\
\hline $1 \mathrm{~A}$ & $206(90.0)$ & $131(90.3)$ & \\
\hline 1B & $23(10.0)$ & $14(9.7)$ & \\
\hline Adjuvant therapy & & & $0.002^{+}$ \\
\hline Observe & $131(57.2)$ & $110(75.9)$ & \\
\hline Vault RT & $94(41.0)$ & $35(24.1)$ & \\
\hline ChemoRT & $1(0.4)$ & $0(0)$ & \\
\hline Direct beam RT & $3(1.3)$ & $0(0)$ & \\
\hline Lymph nodes removed & $27.3 \pm 10.7$ & $24.7 \pm 9.6$ & $0.016^{+}$ \\
\hline Recurrence & $4(1.7)$ & $4(2.8)$ & 0.716 \\
\hline Disease-free interval (mth) & 11.8 & 8.0 & 0.334 \\
\hline Conversion to laparotomy & NA & $5(3.4)$ & \\
\hline Inability to deliver uterus & NA & $1(0.7)$ & \\
\hline Bleeding pedicle & NA & $1(0.7)$ & \\
\hline Dense adhesions & NA & $2(1.4)$ & \\
\hline Severe bronchospasm & NA & $1(0.7)$ & \\
\hline
\end{tabular}

*Endometrioid carcinoma of FIGO Stage 1. tp-value is statistically significant. BMI: body mass index; ChemoRT: combination of adjuvant chemotherapy and radiotherapy; FIGO: International Federation of Gynecology and Obstetrics; NA: not applicable; POD: postoperative day; RT: radiotherapy 
During laparoscopic surgery, the uterus and adnexa were removed through the vagina, which may increase the risk of vaginal cuff recurrence. ${ }^{(14)}$ The rates of vaginal vault recurrence in our study, however, were comparable between the laparoscopic and laparotomy groups.

One limitation of our study was the lack of information on the preoperative comorbid conditions of our patients. This may influence the surgeon's choice of the type of procedure. For patients with previous abdominal operations or intact hymen, laparotomy may be more suitable to remove adhesions and allow for the delivery of the uterus. ${ }^{(4)}$ In addition, given that this was a retrospective study, we were also limited by missing and incomplete data such as the individual tumour grades of the subjects, which is an administrative challenge for most retrospective studies.

In conclusion, laparoscopic surgery is a safe and effective alternative to laparotomy for early stage endometrioid carcinoma, with reduced length of stay, pain score and intraoperative blood loss. The recurrence rate in the laparoscopic surgery group was comparable to that in the laparotomy group. To obtain long-term survival data for further evaluation, studies with a longer follow-up time are required.

\section{REFERENCES}

1. Siegel R, Ma J, Zou Z, Jemal A. Cancer statistics, 2014. CA Cancer J Clin 2014; 64:9-29.

2. Chu LH, Chang WC, Sheu BC. Comparison of the laparoscopic versus conventional open method for surgical staging of endometrial carcinoma. Taiwan J Obstet Gynecol 2016; 55:188-92.
3. Malzoni M, Tinelli R, Cosentino F, et al. Total laparoscopic hysterectomy versus abdominal hysterectomy with lymphadenectomy for early-stage endometrial cancer: a prospective randomized study. Gynecol Oncol 2009; 112:126-33.

4. Chan JK, Gardner AB, Taylor K, et al. Robotic versus laparoscopic versus open surgery in morbidly obese endometrial cancer patients - a comparative analysis of total charges and complication rates. Gynecol Oncol 2015; 139:300-5.

5. Kyrgiou M, Swart AM, Qian W, Warwick J. A comparison of outcomes following laparoscopic and open hysterectomy with or without lymphadenectomy for presumed early-stage endometrial cancer: results from the medical research council ASTEC trial. Int J Gynecol Cancer 2015; 25:1424-36.

6. Bogani G, Cromi A, Serati M, et al. Improving standard of care through introduction of laparoscopy for the surgical management of gynecological malignancies. Int J Gynecol Cancer 2015; 25:741-50.

7. Boggess JF, Gehrig PA, Cantrell L, et al. A comparative study of 3 surgical methods for hysterectomy with staging for endometrial cancer: robotic assistance, laparoscopy, laparotomy. Am J Obstet Gynecol 2008; 199:360.e1-9.

8. Terai $\mathrm{Y}$, Tanaka $\mathrm{T}$, Sasaki $\mathrm{H}$, et al. Total laparoscopic modified radical hysterectomy with lymphadenectomy for endometrial cancer compared with laparotomy. J Obstet Gynaecol Res 2014; 40:570-5.

9. Mourits MJ, Bijen CB, Arts HJ, et al. Safety of laparoscopy versus laparotomy in early-stage endometrial cancer: a randomised trial. Lancet Oncol 2010; 11:763-71

10. Kornblith $A B$, Huang $H Q$, Walker JL, et al. Quality of life of patients with endometrial cancer undergoing laparoscopic international federation of gynecology and obstetrics staging compared with laparotomy: a Gynecologic Oncology Group study. J Clin Oncol 2009; 27:5337-42.

11. Zullo F, Palomba S, Falbo A, et al. Laparoscopic surgery vs laparotomy for early stage endometrial cancer: long-term data of a randomized controlled trial. Am J Obstet Gynecol 2009; 200:296.e1-9.

12. Corrado G, Cutillo G, Pomati G, et al. Surgical and oncological outcome of robotic surgery compared to laparoscopic and abdominal surgery in the management of endometrial cancer. Eur J Surg Oncol 2015; 41:1074-81.

13. Chiou HY, Chiu LH, Chen $\mathrm{CH}$, et al. Comparing robotic surgery with laparoscopy and laparotomy for endometrial cancer management: a cohort study. Int J Surg 2015; 13:17-22.

14. Rabinovich A. Minimally invasive surgery for endometrial cancer. Curr Opin Obstet Gynecol 2015; 27:302-7. 